\title{
Polymer Dynamics: Long Time Simulations and Topological Constraints
}

\author{
Kurt Kremer \\ Max-Planck-Institut für Polymerforschung, Postfach 3148, D-55021 Mainz, Germany
}

\author{
Received on 2 October, 2005
}

\begin{abstract}
Topological constraints, entanglements, dominate the viscoelastic behavior of high molecular weight polymeric liquids. To give a microscopic foundation of the phenomenological tube, recently a method for identifying the so called primitive path mesh that characterizes the microscopic topological state of (computer generated) conformations of long-chain polymer networks, melts and solutions was introduced. Here we give a short account of this approach and compare this to long time simulations.
\end{abstract}

Keywords: Polymer dynamics; Long time simulation; Long-chain polymer networks

\section{INTRODUCTION}

The viscoelastic properties of polymer liquids and their link to the microscopic structure and dynamics are key issues in modern polymer science and biophysics [1-6]. For this a prototypical system is a melt or semi-dilute solution of long (flexible) chain molecules. On a microscopic scale, their Brownian motion is dominated by the restriction that the chains may slide past but not through each other. This introduces motion constraints in a way that each polymer chain can only move along a tube like region around a so called primitive path. This primitive path is the backbone of the appropriately coarse grained chain contour $[7,8]$. Recent, more refined analytical $[3,9]$ and numerical models $[10-14]$ concentrate on the dynamics of these primitive paths. These models are able to quantitatively describe many rheological and single chain dynamics data with a small set of material-specific parameters such as the tube diameter, $d_{T}$. It is the purpose of the present short contribution, to compare a topological approach to determine the tube diameter from chain conformations with long time simulations, which essentially follow an analysis close to experiments.

\section{EXPERIMENTS/SIMULATIONS}

The key quantity of the theory, the mesh of primitive paths or equivalently the tubes with diameter $d_{T}^{2} \sim N_{e}$, the entanglement molecular weight, is experimentally not directly accessible. Thus a variety of experiments have been devised, leading to estimates of $d_{T}$ (or equivalently, $N_{e}$ ), based on different theoretical approximations. Here we discuss three examples.

\section{A. Chain Diffusion $D(N)$}

The diffusion of individual beads as well as of the whole chain can be studied by a variety of experiments as well as computer simulations. Within the concept of the tube or reptation model one expects for the diffusion constant of a whole chain in a melt or semidilute solutions of identical other chains ( $N$ being the chain length):

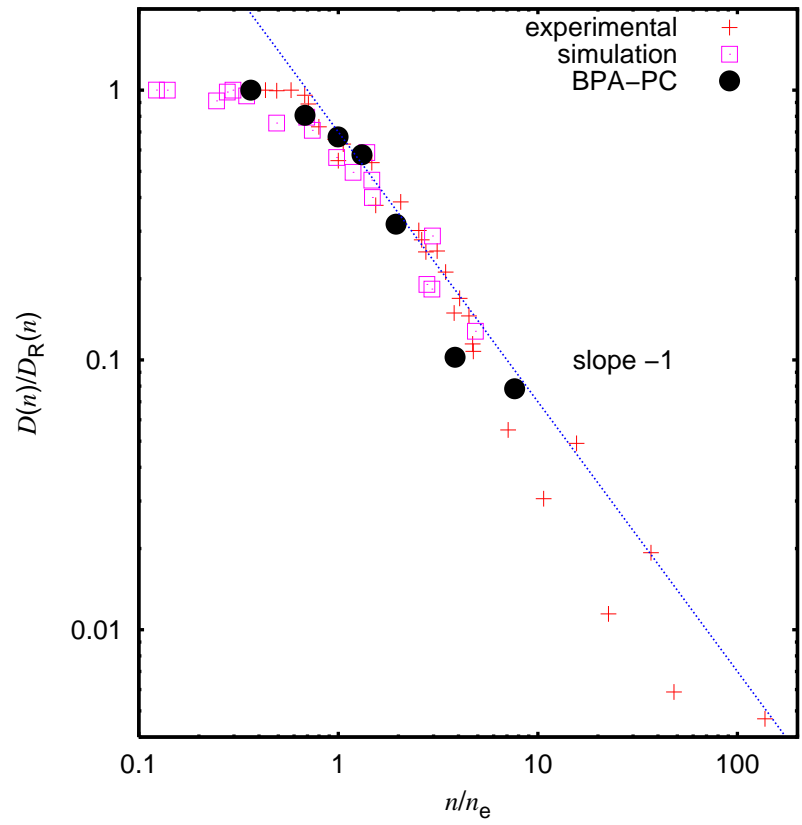

FIG. 1: Scaled diffusion constant $\frac{D(n)}{D_{R}(n)}$ for BPA-PC data and $\frac{D(N)}{D_{R}(N)}$ for the reference data as a function of the scaled chain length $\frac{n}{n_{e}}$ and $\frac{N}{N_{e}}$ respectively from different experimental and simulation results for various polymer systems. The best fit for the entanglement length yields $n_{e}=62 \pm 8$ coarse grained beads, giving approximately $N_{e, D} \simeq$ 15. Figure taken from[16]

$$
D(N) \sim \begin{cases}N^{-1} & ; N \leq N_{e} \\ N_{e} N^{-2} & ; N \gg N_{e}\end{cases}
$$

Recent experiments even suggest a somewhat stronger decay for longer chains[15]. Taking the above relation, we expect a universal curve, when plotting the ratio of $D(N)$ over the hypothetical short chain diffusion constant of the same chain length vs $N / N_{e}$. Using this one can fit a value for $N_{e}$, if a "master plot" is available, cf, Fig. 1. For the present case of the diffusion of polycarbonate, the master curve is from experiments on polyethylene [16]. This allows for a first determination of $N_{e}$ and thus $d_{T}$. 


\section{B. Dynamic Structure Function $S(q, t)$}

Alternatively one can also analyze the dynamic coherent scattering function of individual chains as done for example in neutron scattering experiments. For simple computer polymers, all form factors of the monomers or beads are taken equal and are set to one. Then $S(\mathbf{k}, t)$ reads

$$
S(\mathbf{k}, t)=\frac{1}{N} \sum_{i, j} \exp \left[i \mathbf{k}\left(\mathbf{r}_{i}(t)-\mathbf{r}_{j}(0)\right)\right],
$$

with $\mathbf{r}_{i}(t)$ being the position of bead $i$ at time $t$. For times larger than the time a bead needs to explore the diamter of the tube, but much shorter than the time the chain needs to diffuse out of the tube, the scatterer observes a smeared out bead density within the tube, akin to the effect described by the Debye-Waller factor in solid state physics. This leads in the aforementioned time regime to a plateau in the decay of $S(k, t)[17-19]$

$$
\begin{gathered}
\frac{S(k, t)}{S(k, 0)}=\left\{\left[1-\exp \left[-\left(k d_{T} / 6\right)^{2}\right]\right] f\left(k^{2} b^{2} \sqrt{12 W t / \pi}\right)\right. \\
\left.\quad+\exp \left[-\left(k d_{T} / 6\right)^{2}\right]\right\} \frac{8}{\pi^{2}} \sum_{p=1, o d d}^{\infty} \frac{\exp \left[-t p^{2} / \tau_{d}\right]}{p^{2}}
\end{gathered}
$$

where $f(u)=\exp \left[u^{2} / 36\right] \operatorname{erfc}(u / 6), b$ is the bond length and $W$ is the bead friction. Figure ?? shows this, again for the example of polycarbonates[16]. From the above expression $d_{T}$ can be estimated. Again, $d_{T}$ is used to fit the data to the theory. If we assume that $d_{T}^{2}=R^{2}\left(N_{e}\right)=N_{e} c_{\infty} b^{2}, R^{2}\left(N_{e}\right)$ being the mean square end to end distance of a chain of $N_{e}$ beads and $c_{\infty}$ the Flory characteristic ratio [20], it is then possible to calculate the entanglement length $N_{e}$.

\section{Plateau Modulus $G_{N}^{0}$}

The third approach goes back to the very origin of the tube models and employs the analogy between long chain polymer melts and networks. Under elongational or shear strain the initial stress relaxes rapidly towards a plateau $G_{N}^{0}$. In a simulation, the normal stress $\sigma_{n}$ can be determined by the microscopic virial tensor and the resulting stress plateau can be fitted to the stress strain formulas from classical rubber elasticity (CRE) (e.g. elongational strain in the limit of small deformation $\lambda)[21,22]$

$$
\sigma_{n}=G\left(\lambda^{2}-\frac{1}{\lambda}\right)
$$

to determine $G_{N}^{0}$, which in turn is related to $N_{e}[1,3]$ by the expression

$$
G_{N}^{0}=\frac{4}{5} \frac{\rho k_{B} T}{N_{e}}
$$

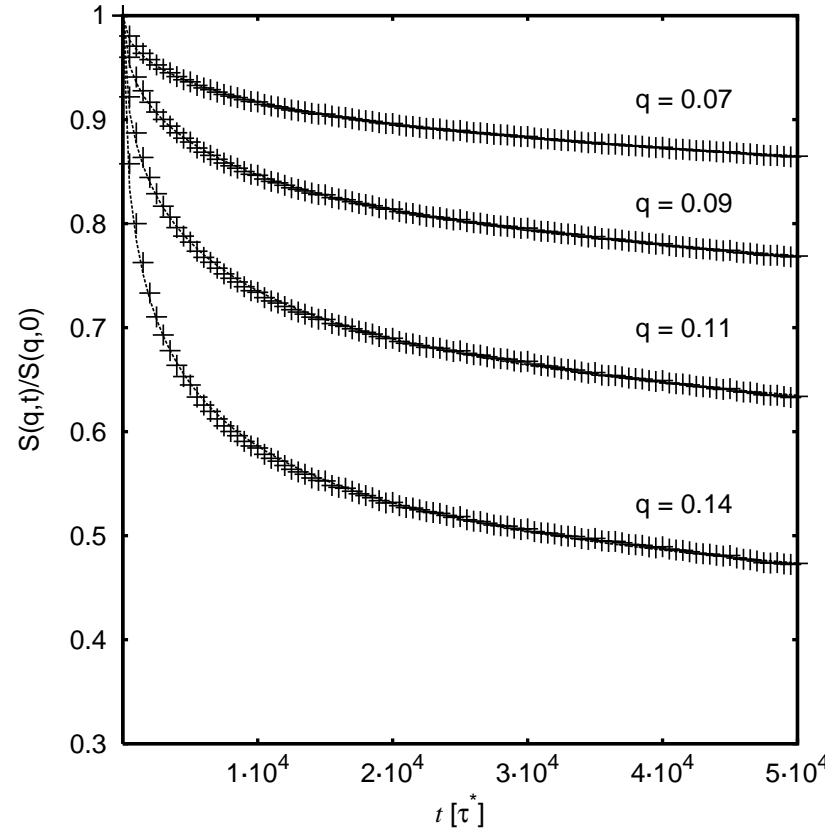

FIG. 2: Scaled dynamic structure factor $S(q, t) / S(q, 0)$ as a function of time for the polycarbonate melt with chain length $N=120$ repeat units. For each $q$-value, dotted lines show the fit to the expression of equation 3. Figure taken from[16]

TABLE I: Values obtained for $N_{e}$ using the different approaches discussed in the text for fully flexible bead spring chains [17] and a coarse grained model of polycarbonate (BPA-PC) $[16,30]$. The maximum chain lengths studied are also indicated.

\begin{tabular}{|c|c|c|}
\hline & $N_{e}^{B P A-P C}$ & $N_{e}^{\text {bead-spring }}$ \\
\hline$D(N)$ & $\approx 15$ & $\approx 70$ \\
$S(q, t)$ & 10 & 55 \\
$G_{N}^{0}$ & $5-6$ & 72 \\
$N_{\max }$ & 120 & 2000 \\
\hline
\end{tabular}

where $\rho$ is the bead density. This is the most direct experimental way to determine $d_{T}$ and $N_{e}$ respectively, as no fitting is required and only the underlying theory is applied. However, this again is not a direct measurement of $N_{e}$.

In Table I the outcome of the different approaches from simulations of a special coarse grained model of polycarbonate and for highly flexible simple bead spring chains are given. For both cases the material specific quantity $N_{e}$ is very different, if obtained by different methods. While this by itself is not surprising and problematic, it is the fact that the ratio of the estimates from individual "experiments" is very different for the two different cases. Thus there is a clear need for an independent ansatz to obtain the material specific quantitiy $N_{e}$, without referring to the rather indirect experimental approaches. 


\section{PRIMITIVE PATH ANALYSIS}

To do this, we go back to the original idea of Edwards [23] who identified the random walk-like axis of the tube with what he called the "primitive" path: the shortest path between the endpoints of the original chain into which its contour can be contracted without crossing any obstacle. Similar to the tube, the primitive path is usually discussed without specifying the relation between the obstacles and the melt structure. However the obstacles themselves are chains, which has to be taken into account, when constructing the primitive path [24-26]. In Ref. [24] we introduced a primitive path analysis (PPA) where all polymers in the system are contracted simultaneously in a way, that a linear stress strain relationship is kept. This allows us to establish the microscopic foundations of the tube model and to endow a highly successful phenomenological model with predictive power for structure-property relations.

We performed the primitive path analysis on a variety of different simple bead spring chain melts [27] and semi dilute solutions [28] with variable intrinsic stiffness [29]. All these cases include the crucial ingredients characteristic for polymeric systems: connectivity, flexibility, local liquid-like monomer packing and mutual uncrossability of the chain backbones. The parameters used for the PPA are given in [25].

Monodisperse polymer melts of $M=200-500$ chains of length $50 \leq N \leq 700$ at a bead density of $\rho=0.85 \sigma^{-3}$ (in Lennard Jones units) are studied. By introducing a small intrinsic bond bending potential, the Kuhn length, $l_{K}=\left\langle R^{2}\right\rangle / L$, is varied between $1.80 \sigma$ and $3.34 \sigma .\left\langle R^{2}\right\rangle$ is the mean squared end to end distance of the chain and $L$ is the chain contour length. For details see Ref. [29]. In addition, we present results for a coarse-grained model for polycarbonate (BPAPC) $[16,30]$. In this case, we analyzed melt configurations for $M=100$ chains of $N=60$ chemical repeat units which are represented by four beads each. All the melt samples studied are tabulated in [25].

The implementation of the PPA is straightforward: First the chain-ends are fixed in space. Then, all intrachain interactions other than the FENE bond interaction, which has its minimum at $r=0$, are switched off. Finally the total energy is minimized by cooling the system slowly to $T \rightarrow 0$. Without thermal fluctuations and intra-chain EV interactions, the bond springs try to reduce the bond length to zero and pull the chains taut. The interchain EV interactions provide an energy barrier to prevent chain crossings. Needless to say, a crucial ingredient for the success of the whole procedure is the availability of well equilibrated conformations[29, 31]! For details of the PPA protocol we refer to [25]. The algorithm introduced so far does not account for self-entanglements. In a recent variant of the approach this is included in the PPA [25].

\section{RESULTS - COMPARISON TO EXPERIMENTS}

In order to understand the relationship between the melt structure and properties like the plateau modulus for many different polymers and simple model systems we need a dimensionless way to compare data. To render the $G_{N}^{0}$ values dimensionless, we need an energy scale and a length scale. As the dominant contribution to $G_{N}^{0}$ is of entropic origin, $k_{B} T$ as the energy scale suggests itself. However, there are essentially two independent length scales that characterize the local structure of polymer melts. One, the Kuhn length, $l_{K}[1]$ is defined for random walks as the length of an individual step of a freely jointed chain with the same mean-square end-toend distance $\left\langle R^{2}\right\rangle=l_{K} L$ and contour length $L$. Random walks however do not densely fill space. Locally however, most monomers will belong to the same chain. The length at which the polymers start to interpenetrate is given by the packing length, $p=\left(\rho_{\text {chain }}\left\langle R^{2}\right\rangle\right)^{-1}$. The packing length can be visualized as the average strand strand distance. It is relevant to note that the product of the number density of chains, $\rho_{\text {chain }}$, and of $\left\langle R^{2}\right\rangle$ is independent of chain length for a fixed monomer number density, $\rho$. Following the standard convention [32] based on the chemical structure of the polymers, we choose $l_{K}$ as the unit of length. With this choice, instead of $G_{N}^{0}$ we can consider the dimensionless quantity $G_{N}^{0} l_{K}^{3} / k_{B} T$. This quantity has to be a function of the only remaining dimensionless parameter: the ratio of Kuhn and packing length $l_{K} / p$.

The available experimental data for dense melts are the result of a long term substantial experimental effort [33, 34]. Rheological measurements were performed on these samples and in many cases the $\left\langle R^{2}\right\rangle$ values were determined using small angle neutron scattering. Refs. [33, 34] provide the values for the plateau modulus, $G_{N}^{0}$, the mass density, $\rho_{m}$, the ratio of the mean-square end-to-end distance to the molecular weight of the polymer, $\left\langle R^{2}\right\rangle / M$, and the packing length, $p=M /\left(\left\langle R^{2}\right\rangle \rho_{m} N_{A}\right)$, where $N_{A}$ is the Avagadro number. All the melt data points obey the empirical relation $G_{N}^{0}=0.00226 k_{B} T / p^{3}$, indicated as the dashed line in Fig. 3. The way to determine $l_{k}$ for experimental systems, depends on the definition of the contour length. It is however worthwhile to note, if the empirical relation is valid, then any choice for $l_{K}$ will preserve the scaling relationship between $G_{N}^{0}$ and $p$. The actual choice for $l_{K}$ will determine the position of the data points in Fig. 3 and will just move them along the dashed line in the figure. All the melt data shown in the figure are tabulated in [25], where also a more detailed discussion of the mapping and the length scales can be found.

The aforementioned mapping can be also be used to identify bead spring polymer models to individual chemical species. The standard model [27] with fully flexible polymer chains used in molecular dynamics simulations corresponds to $l_{K} / p=2.68$. Among the available experimental data, the chemical species with the closest $l_{K} / p$ value is natural rubber (cis-Polyisoprene) with $l_{K} / p=2.72$. This suggests that the elastic properties of the usually studied bead spring polymer model corresponds most closely to that of natural rubber, the prototypical experimental system for elastic behavior.

\section{DISCUSSION}

Having provided a method to determine the primitive paths for melt configurations of entangled bead-spring model polymers, we can now test the predictive power of the tube model. 


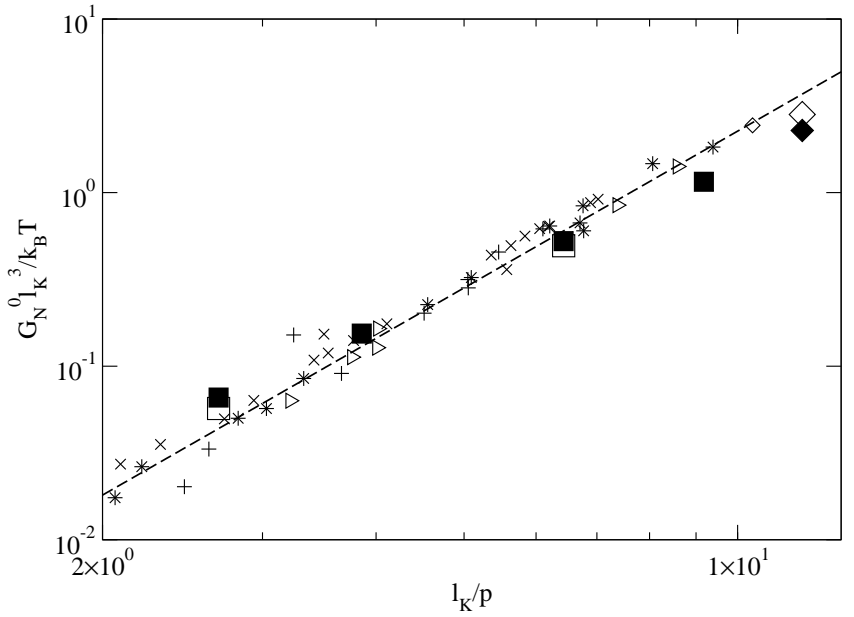

FIG. 3: Dimensionless plateau moduli $G_{N}^{0} l_{K}^{3} / k_{B} T$ as a function of the dimensionless ratio $l_{K} / p$ of Kuhn length $l_{K}$ and packing length $p$. Inset (a) contains (i) experimentally measured plateau moduli for polymer melts[? ] (* Polyolefins, $\times$ Polydienes, + Polyacrylates and $\triangleright$ miscellaneous, (ii) plateau moduli inferred from the normal tensions measured in computer simulation of bead-spring melts [17, 24] $(\square)$ and a semi-atomistic polycarbonate melt [16] $(\diamond)$ under an elongational strain, and (iii) predictions of the tube model Eq. 6 based on the results of our PPA for bead-spring melts $(\boldsymbol{\square})$, and the semiatomistic polycarbonate melt $(\diamond)$. The line indicates the best fit to the experimental melt data for polymer melts by Fetters et al. [34]. Errors for all the simulation data are smaller than the symbol size.
To this end, we use the standard expression $[1,24]$

$$
G_{N}^{0}=\frac{4}{5} \frac{k_{B} T}{p d_{T}^{2}}=\frac{4}{5} \frac{\rho k_{B} T}{N_{e}}
$$

which relates the plateau modulus to the Kuhn length of the primitive path. The $d_{T}$ values can be obtained from the contour length of the primitive path, $L_{p p}$ (which is the sum of the lengths of all the back bone bonds of a chain averaged over all the chains in the melt) using $d_{T}=\left\langle R^{2}\right\rangle / L_{p p}$. For details refer [24, 25]. Fig. 3 shows an explicit comparison of the dimensionless plateau moduli $G_{N}^{0} l_{K}^{3} / k_{B} T$ of experimental systems and bead-spring model polymers with identical ratios $l_{K} / p$. The good agreement of the two data sets confirms the insensitivity of entanglement effects to atomistic details and provides the necessary validation of our generic bead-spring models.

\section{ACKNOWLEDGEMENTS}

The present results are a subset of those, which are originating from a very fruitful and (in some cases) very longstanding collaboration with G. S. Grest, R. Everaers, S. Sukumaran, C. Svaneborg, L. Delle Site and S. Leon. The present work is updated and shortened version of Ref. [35].
[1] M. Doi and S. F. Edwards, Theory of Polymer Dynamics, Clarendon: Oxford 1986.

[2] H. Watanabe, Prog. Pol. Sci. 24, 1253 (1999).

[3] T. C. B. McLeish, Adv. Phys. bf 5, 1379 (2002).

[4] D. Humphrey, C. Duggan, D. Smith, and J. Käs, Nature 416, $413(2002)$

[5] T. T. Perkins, D. E. Smith, and S. Chu, Science 264, 819 (1994).

[6] R. B. Bird, R. C. Armstrong, and O. Hassager, Dynamics of Polymeric Liquids, Vol. 1, Wiley: New York (1977).

[7] S. F. Edwards, Proc. Phys. Soc. 92, 9 (1967).

[8] P. G. J. de Gennes, J. Chem. Phys. 55, 572 (1971).

[9] A. E. Likhtman, T. C. B. McLeish, Macromolecules 356332 (2002).

[10] Y. Masubuchi, J.-I. Takimoto, K. Koyama, G. Ianniruberto, G. Marrucci, and F. Greco, J. Chem. Phys. 1154387 (2001).

[11] J. T. Padding, W. J. Briels, J. Chem Phys. 117, 925 (2002).

[12] K. Iwata, M. Tanaka, N. Mita, and Y. Kohno, Polymer 43, 6595 (2002).

[13] M. Doi, J. Takimoto, Phil. Trans. R. Soc. Lond. A 361, 641 (2003).

[14] J. D. Schieber, J. Neergaard, and S. Gupta, J. Rheol. 47, 213 (2003).

[15] T. P. Lodge, Phys. Rev. Lett. 83, 3218 (1999).

[16] S. Leon, L. D. Site, N. van der Vegt, and K. Kremer, Macromolecules 38, 8078 (2005).

[17] M. Puetz, K. Kremer, and G. S. Grest, Europhys. Lett. 49, 735 (2000).

[18] P. G. de Gennes, J. Phys. (Paris) 42, 735 (1981).

[19] P. Schleger, B. Farago, C. Lartigue, A. Kollmar, and D. Richter,
Phys. Rev. Lett. 81, 124 (1998).

[20] P. Flory, Statistical Mechanics of Chain Molecules, Interscience: New York (1969).

[21] L. R. G. Treloar, The Physics of Rubber Elasticity, Clarendon Press: Oxford (1975).

[22] J. D. Ferry, Viscoelastic Properties of Polimers, Wiley: New York (1980).

[23] S. F. Edwards, Brit. Polym. J. 9, 140 (1977).

[24] R. Everaers, S. K. Sukumaran, G. S. Grest, C. Svaneborg, A. Sivasubramanian, and K. Kremer, Science 303, 823 (2004).

[25] S. K. Sukumaran, G. S. Grest, K. Kremer, and R. Everaers, J. Polym. Sci. Part B: Polym. Phys. Ed., to be published (2004)

[26] M. Rubinstein, E. Helfand, J. Chem. Phys. 82, 2477 (1985).

[27] K. Kremer, G. S. Grest, J. Chem. Phys. 92, 5057 (1990).

[28] P. Ahlrichs, R. Everaers, B. Duenweg, Phys. Rev. E 64, 040501 (2001).

[29] R. Auhl, R. Everaers, G. S. Grest, K. Kremer, and S. J. Plimpton, J. Chem. Phys. 119, 12718 (2003).

[30] C. F. Abrams, K. Kremer, Macromolecules 36, 260 (2003).

[31] R. S. Hoy, M. O. Robbins, Phys. Rev. E 72, 061802 (2005).

[32] W. W. Graessley, S. F. Edwards, Polymer 22, 1329 (1981).

[33] L. J. Fetters, D. J. Lohse, D. Richter, T. A. Witten, and A. Zirkel, Macromolecules 27, 4639 (1994).

[34] J. Fetters, L. D. Johse, and W. W. Graessley, J. Polym. Sci. B: Polymer Phys. 37, 1023 (1999).

[35] K. Kremer, S. K. Sukumaran, R. Everaers, and G. S. Grest, Comp. Phys. Comm. 169, 75 (2005). 